Tropical Journal of Pharmaceutical Research March 2020; 19 (3): 549-555

ISSN: $1596-5996$ (print); 1596-9827 (electronic)

(C) Pharmacotherapy Group, Faculty of Pharmacy, University of Benin, Benin City, 300001 Nigeria.

\title{
Aqueous leaf extract of Sutherlandia frutescens attenuates ROS-induced apoptosis and loss of mitochondrial membrane potential in MPP+-treated SH-SY5Y cells
}

\author{
Adaze B Enogieru, Sylvester I Omoruyi, Okobi E Ekpo* \\ Department of Medical Biosciences, University of the Western Cape, Robert Sobukwe Road, Private Bag X17, Bellville 7535, \\ South Africa
}

*For correspondence: Email: oekpo@uwc.ac.za; Tel: +27 (0)21959 3962

Sent for review: 12 December 2019

Revised accepted: 26 February 2020

\begin{abstract}
Purpose: To investigate the neuroprotective activity of the aqueous extract of Sutherlandia frutescens (SF) against 1-methyl-4-phenylpyridinium $\left(\mathrm{MPP}^{+}\right)$-induced toxicity in SH-SY5Y neuroblastoma cells. Methods: SH-SY5Y neuroblastoma cells were divided into different treatment groups: untreated cells, cells treated with MPP ${ }^{+}$alone $(2 \mathrm{mM})$, cells pretreated with SF $(20 \mu \mathrm{g})$ prior to MPP ${ }^{+}(2 \mathrm{mM})$ treatment and cells treated with SF $(20 \mu \mathrm{g})$ alone. Twenty-four hours after treatment with MPP ${ }^{+}$, cell viability was assessed by MTT assay, and changes in cell morphology, intracellular reactive oxygen species (ROS) production, mitochondrial membrane potential (MMP) as well as caspases $3 / 7$ and 9 activities were determined.

Results: Treatment of SH-SY5Y cells with MPP ${ }^{+}$alone significantly altered cellular morphology, increased ROS production ( $p=0.005)$, induced a significant loss of MMP $(p=0.0011)$ and caused significant apoptotic cell death, via the activation of caspases $3 / 7$ and 9 ( $p \leq 0.0359)$. These effects were however significantly $(p \leq 0.0359)$ attenuated in cells pre-treated with the aqueous leaf extract of SF, indicating the possible neuroprotective activity of the SF extract.

Conclusion: The results of this study suggest that the aqueous leaf extract of SF may be neuroprotective against MPP+-induced toxicity via apoptotic cell death and inhibition of ROS production. Further mechanistic studies are required to validate the results of the present study using additional PD models, different extract preparations and active compounds derived from SF.
\end{abstract}

Keywords: Parkinson's disease, $\mathrm{MPP}^{+}$, Sutherlandia frutescens, Reactive oxygen species, Apoptosis, Neurodegeneration

\begin{abstract}
This is an Open Access article that uses a fund-ing model which does not charge readers or their institutions for access and distributed under the terms of the Creative Commons Attribution License (http://creativecommons.org/licenses/by/4.0) and the Budapest Open Access Initiative (http://www.budapestopenaccessinitiative.org/read), which permit unrestricted use, distribution, and reproduction in any medium, provided the original work is properly credited.
\end{abstract}

Tropical Journal of Pharmaceutical Research is indexed by Science Citation Index (SciSearch), Scopus, International Pharmaceutical Abstract, Chemical Abstracts, Embase, Index Copernicus, EBSCO, African Index Medicus, JournalSeek, Journal Citation Reports/Science Edition, Directory of Open Access Journals (DOAJ), African Journal Online, Bioline International, Open-J-Gate and Pharmacy Abstracts

\section{INTRODUCTION}

Parkinson's disease (PD) is the second most common, age-dependent neurodegenerative disorder resulting from continuous loss of dopaminergic neurons in the pars compacta of the substantia nigra of the midbrain (SNpc). Resting tremor, bradykinesia and muscle rigidity are some of the motor symptoms associated with PD, while cognitive and neuropsychiatric disorders constitute the non-motor symptoms [1]. There is currently no cure for PD, hence the 
search for effective treatment options capable of halting the death of dopaminergic neurons with little or no side effects, is plausible.

In order to study the mechanisms involved in PD, the standard cellular model of the disease was induced using the neurotoxic compound 1methyl-4-phenylpyridinium $\left(\mathrm{MPP}^{+}\right)$, an active metabolite of 1-methyl-4-phenyl-1,2,3,6tetrahydropyridine (MPTP) was used. When $\mathrm{MPP}^{+}$is taken up by the dopamine transporter, it causes damage to dopaminergic neurons in a way that is comparable to the mechanisms of the human disease [2]. For instance, $\mathrm{MPP}^{+}$inhibits complex I of the mitochondrial electron transport chain (ETC), thus diminishing the production of ATP and increasing the formation of reactive oxygen species (ROS) [2].

Although the etiology and exact molecular mechanisms involved in the neuronal degeneration observed in PD are poorly understood, the involvement of oxidative stress (OS), apoptosis and mitochondrial dysfunction have been reported. Over the years, research has focused more on the pathological importance of OS in PD, owing to its role in aging and agingrelated diseases in general. Oxidative stress is known to be caused by the imbalance in the production of ROS and antioxidants and is believed to be a key mechanism that heralds and initiates the death of neurons in PD [3].

Earlier studies have shown substantial evidence of oxidative stress in the brains of animal models of PD [4]. Oxidants are by-products of oxidative phosphorylation, thus making mitochondria the major sites of ROS generation within the cell. In most neurodegenerative diseases characterized by mitochondrial respiratory defects, including PD, the ROS generated by the ETC increases and overwhelms the antioxidant protection mechanisms. This is evidenced by post-mortem reports of complex I deficiency in the SN of individuals with $P D$, thus establishing a direct link between ROS, mitochondrial dysfunction and the disease [5].

Similarly, the vast majority of in vivo and in vitro models of PD have been reported to show morphological apoptosis and activation of caspases [6]. In most of these models, the involvement of apoptotic signalling pathways and the attenuation of cell death by caspase inhibitors tend to support suggestions of apoptosis as a mechanism of cell death in PD. Thus, it is plausible to investigate antioxidant-rich medicinal plants for their apoptosis-inhibiting effects as well as their ability to mitigate mitochondrial dysfunction and ROS production in dopaminergic neurons. Plant extracts with such properties could provide potential sources of novel therapeutic agents for the protection or treatment of PD.

Sutherlandia frutescens (SF), also known as 'cancer bush', is a versatile medicinal plant commonly used in South Africa [7]. Its decoctions have been used for many years by traditional healers to treat open wounds, inflammation, fever, eye infections, chicken pox and haemorrhoids [8]. It is also consumed as a herbal remedy by individuals living with HIVIAIDS in South Africa as an immune-booster and to improve general well-being [9].

Various studies have investigated the beneficial effects of SF in cancer [10], diabetes [11] and inflammation [12]. Phytochemical evaluations of this plant have shown that it contains significant amounts of pharmacologically important constituents, including gamma-aminobutyric acid, glycosides, saponins, and L-canavanine. Although SF has been reported to show activity in various disease models, studies on its neuroprotective activity in a Parkinson's disease model have not been reported. This study therefore investigated the mechanisms by which SF could potentially provide neuroprotection against $\mathrm{MPP}^{+}$-induced cell death in the $\mathrm{SH}-\mathrm{SY} 5 \mathrm{Y}$ human neuroblastoma cell line used as a model of PD.

\section{EXPERIMENTAL}

\section{Preparation of extract}

Dry-milled leaf powder of SF (variety Incana E. Mey.) [Family: Fabaceae] was purchased from Big Tree Health Products (Fish Hoek, South Africa). To prepare the aqueous extract of the plant, $1 \mathrm{~kg}$ of the leaf powder was added to boiling water and incubated overnight. The extract was then filtered with Whatman filter paper and freeze-dried to yield the aqueous extract which was stored at $-20^{\circ} \mathrm{C}$ until needed.

\section{Cell culture maintenance}

SH-SY5Y cells were maintained in Dulbecco's modified Eagle medium (DMEM) (Life Technologies, USA), supplemented with $10 \%$ Fetal bovine serum (FBS) (Life Technologies, USA) and $1 \%$ penicillin-streptomycin (Lonza, USA), at $37{ }^{\circ} \mathrm{C}, 5 \% \mathrm{CO}_{2}$ in a humidified incubator. The culture medium was changed every two days and the cells were trypsinized with $1 \mathrm{X}$ trypsin-versene EDTA mixture (Lonza, USA) and transferred to a different dish for 
regrowth once a confluency of 70 - $80 \%$ was reached.

Cytotoxicity screening, cell viability and toxicity assay

Cytotoxicity screening was performed to ensure that SH-SY5Y cells were exposed to the correct concentrations of $\mathrm{MPP}^{+}$and SF. A fresh stock concentration of $\mathrm{MPP}^{+}$was prepared on the day of the experiment and dilutions were made up for final concentration ranges of $0-4000 \mu \mathrm{M}$. For SF, a fresh stock concentration was prepared on the day of the experiment and dilutions were made up for final concentration ranges of $0-60$ $\mu \mathrm{g}$. Cell viability was determined using MTT [3(4,5-dimethylthiazol-2-yl)-2,5-diphenyltetrazolium bromide, St. Louis, MO, USA] assay [13, 14]. SH-SY5Y cells, seeded at a density of $1.0 \times 10^{4}$ cells/well in 96-well plates, were allowed to attach for $24 \mathrm{~h}$. Based on the findings from the cytotoxicity screening, cells were pretreated with $20 \mu \mathrm{g}$ of SF for $1 \mathrm{~h}$ and then treated with 2000 $\mu \mathrm{M}$ of $\mathrm{MPP}^{+}$for $24 \mathrm{~h}$. Thereafter, $20 \mu \mathrm{L}$ of the MTT solution was added to each well of the plate and incubated for $3 \mathrm{~h}$. Absorbance was measured at a wavelength of $560 \mathrm{~nm}$ using the Glomax Multi Detection System (Promega, USA).

The trypan blue (TB) dye exclusion assay was used to measure toxicity in treated and untreated samples. The TB dye only enters non-viable cells through their damaged cell membranes while healthy viable cells exclude this dye. For this assay, the SH-SY5Y cells were seeded at a density of $1.1 \times 10^{5} / \mathrm{ml}$ in $60 \mathrm{~mm}$ dishes and treated as previously described. Adherent cells were detached by trypsinization, pelleted and resuspended in fresh culture media. A $20 \mu \mathrm{L}$ of the cell suspension was added to an equivalent volume of $0.4 \%$ trypan blue dye (Sigma Aldrich, St. Louis, MO, USA) and loaded into a BioRad TC20 ${ }^{\mathrm{TM}}$ automated cell counter. Toxicity was expressed as the percentage of dead cells relative to the total cell count.

\section{Morphological evaluation of cells}

The SH-SY5Y cells were seeded at a density of $1.1 \times 10^{5} / \mathrm{mL}$ in $60 \mathrm{~mm}$ dishes and were allowed to attach for $24 \mathrm{~h}$. The cells were treated as earlier described and morphological changes were observed using a ZEISS Primo Vert (Germany) light microscope.

\section{Determination of reactive oxygen species production}

The production of reactive oxygen species was determined using the 2',7'-dichlorofluorescin diacetate (DCFH-DA, St. Louis, MO, USA) dye. The SH-SY5Y cells were seeded at a density of $1.2 \times 10^{5} / \mathrm{mL}$ in $100 \mathrm{~mm}$ dishes and treated as previously described. After treatment, adherent cells were dislodged with a scrapper and spun using a Bio-Rad tabletop centrifuge at $3000 \mathrm{rpm}$ for $5 \mathrm{~min}$. Cell pellets were resuspended and incubated with $25 \mu \mathrm{M}$ of DCFH-DA for $45 \mathrm{~min}$. Stained cells were pelleted, resuspended in PBS $(500 \mu \mathrm{L})$ and thereafter analysed on an Accuri flow cytometer (BD Biosciences Pharmingen, San Diego, CA, USA), with a total of 10000 events acquired for each sample.

\section{Determination of mitochondrial membrane potential}

Mitochondrial membrane potential was determined using rhodamine 123 (St. Louis, MO, USA) fluorescence dye. The SH-SY5Y cells were seeded at a density of $1.2 \times 10^{5} / \mathrm{mL}$ and were treated as previously described. Thereafter, adherent cells were dislodged with a cell scraper and spun at $3000 \mathrm{rpm}$ for $5 \mathrm{~min}$. Cell pellets were resuspended and incubated with $10 \mu \mathrm{M}$ of rhodamine123 for $30 \mathrm{~min}$. Stained cells were pelleted, resuspended in PBS $(500 \mu \mathrm{L})$ and thereafter analysed on an Accuri flow cytometer (BD Biosciences Pharmingen, San Diego, CA, USA), with a total of 10000 events acquired for each sample.

\section{Evaluation of Caspase $3 / 7$ and Caspase 9 activities}

The ApoTarget ${ }^{\mathrm{TM}}$ Caspase- 9 Protease Assay (Life Technologies, USA) was used to evaluate the activity of caspase-9. SH-SY5Y cells were seeded at a density of $1.2 \times 10^{5}$ cells $/ \mathrm{mL}$ in 100 $\mathrm{mm}$ dishes and treated as earlier described. Adherent cells were displaced, pelleted, resuspended in lysis buffer and incubated on ice for $10 \mathrm{~min}$. Experiments were performed according to the manufacturer's instructions, and absorbance was detected using a Polarstar Omega plate reader (BMG Labtech, USA) at 405 $\mathrm{nm}$. Also, the Caspase $3 / 7$ assay kit (Promega, USA) was used to evaluate the activity of caspase $3 / 7$. SH-SY5Y cells were seeded at a density of $1.0 \times 10^{4}$ cells/well in white-walled 96well plates and treated as earlier described. After treatment, experiments were performed according to the manufacturer's instructions, and luminescence was measured using the Glomax Multi Detection System.

\section{Statistical analysis}

Statistical analyses were done using the $\begin{array}{llll}\text { GraphPad } & \text { Prism } & \text { Software } & \text { V7 }\end{array}$ 
(www.graphpad.com/scientific-software/prism/), and data expressed as mean with standard error of mean (SEM) from three independent experiments. One-way analysis of variance (ANOVA) followed by Tukey's multiple comparisons post-hoc test was used to analyze the relationship between variables. Significance was considered at $p<0.05$.

\section{RESULTS}

\section{Dose-response of $\mathrm{MPP}^{+}$and SF toxicity.}

Cell viability significantly decreased following a 24-h incubation of the SH-SY5Y cells with increasing concentrations of $\mathrm{MPP}^{+}(0-4000$ $\mu \mathrm{M})$. The dose-response investigation was done to determine the $\mathrm{MPP}^{+}$concentration which will result in approximately $50 \%$ viable cells (Figure $1 \mathrm{~A})$. For SF, there was a significant increase in cell viability after $24-h$ treatment with increasing concentrations of the SF extract $(20-60 \mu \mathrm{g})$. Consequently, a concentration of $20 \mu \mathrm{g}$ was chosen for use in further experiments based on the highest absorbance value $(p=0.0008$, Figure $1 \mathrm{~B})$.

\section{Effect of SF on $\mathrm{MPP}^{+}$-induced toxicity in SH- SY5Y cells}

The MTT assay results (Figure $1 \mathrm{C}$ ) showed that treatment with $\mathrm{MPP}^{+}$alone caused a significant decrease in cell viability $(p<0.0001)$, while pretreatment with SF before exposure to $\mathrm{MPP}^{+}$ resulted in significant increase $(p=0.0008$,$) in$ cell viability. In the SH-SY5Y cells treated with SF alone, cell viability was not significantly different ( $p=0.4204)$ when compared to the viability of the untreated cells. Similarly, results of the trypan blue dye exclusion assay revealed that treatment with $\mathrm{MPP}^{+}$alone caused a significant increase in cell toxicity $(p=0.0004)$, while pretreatment with SF significantly decreased ( $p=0.0016$, Figure $1 \mathrm{D}$ ) cell toxicity. When compared to the untreated SH-SY5Y cells, the toxicity observed in SH-SY5Y cells treated with SF alone was not statistically significant $(p=$ 0.9511).

\section{Morphology of SH-SY5Y cells following SF pretreatment}

Morphological evaluation of the SH-SY5Y cells treated with $\mathrm{MPP}^{+}$alone showed shrinkage and rounding up of cell bodies while SF pre-treatment appeared to have prevented these $\mathrm{MPP}^{+}$induced morphological alterations. On the other hand, the morphology of cells exposed to SF alone appeared similar to that of the untreated cells (Figure 2).
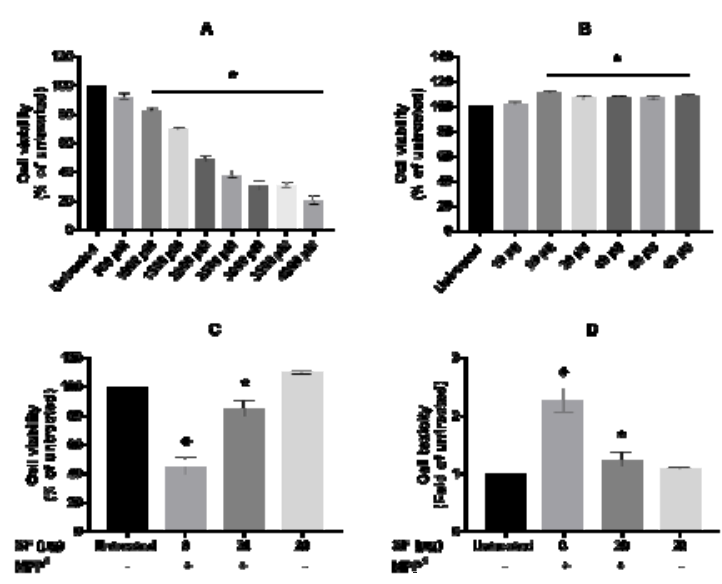

Figure 1: Effect of treatment with SF extract on $\mathrm{MPP}^{+}$induced toxicity in SH-SY5Y cells. (A) MPP ${ }^{+}$toxicity screening (B) SF toxicity screening (C) MTT cell viability assay (D) Trypan blue dye exclusion assay. $\bullet p<0.0005$ vs untreated SH-SY5Y cells; ${ }^{*} p<0.05$ vs SH-SY5Y cells treated with MPP ${ }^{+}$only

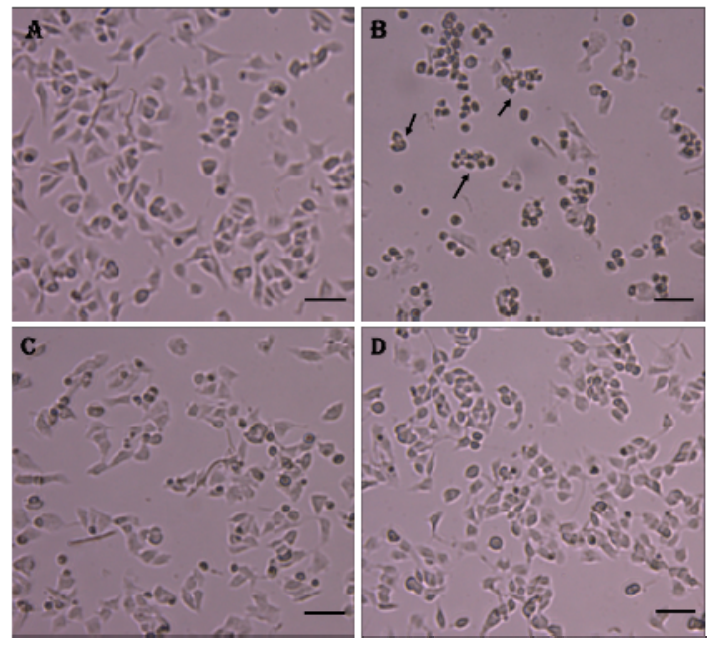

Figure 2: Effects of treatment with SF extract on $\mathrm{MPP}^{+}$-induced morphological alterations in SH-SY5Y cells. (A) Untreated SH-SY5Y cells (B) SH-SY5Y cells treated with $\mathrm{MPP}^{+}$alone $(\mathrm{C}) \mathrm{SH}-\mathrm{SY} 5 \mathrm{Y}$ cells pretreated with SF and then treated with $\mathrm{MPP}^{+}$(D) SH-SY5Y cells treated with SF only. Arrows indicate shrinking and contracting SH-SY5Y cells. Scale bar: $5 \mu \mathrm{m}$

\section{ROS production following SF pretreatment}

Whereas ROS levels significantly increased $(p=$ 0.005) in SH-SY5Y cells treated with $\mathrm{MPP}^{+}$ alone, pretreatment with SF significantly reduced ( $p=0.0316)$ ROS production. However, there was no significant difference $(p=0.994)$ in the ROS levels in the SH-SY5Y cells treated with SF alone when compared to the untreated cells (Figure 3). 

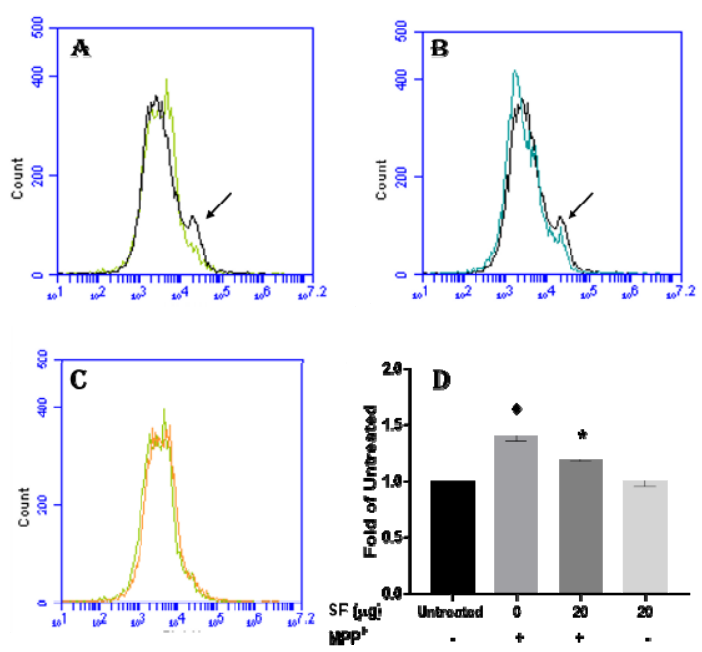

Figure 3: Effects of treatment with SF extract on $\mathrm{MPP}^{+}$-induced ROS production. (A) Untreated vs $\mathrm{MPP}^{+}$alone (B) SF pretreated vs $\mathrm{MPP}^{+}$alone (C) Untreated vs SF alone (D) Bar chart showing the effect of SF on ROS production. Arrows indicate fluorescence intensity of cells treated with $\mathrm{MPP}^{+}$only. $\bullet p<0.0005$ vs untreated SH-SY5Y cells; ${ }^{*} p<0.05$ vs SH-SY5Y cells treated with MPP ${ }^{+}$only

Mitochondrial membrane potential in SHSY5Y cells following SF pretreatment
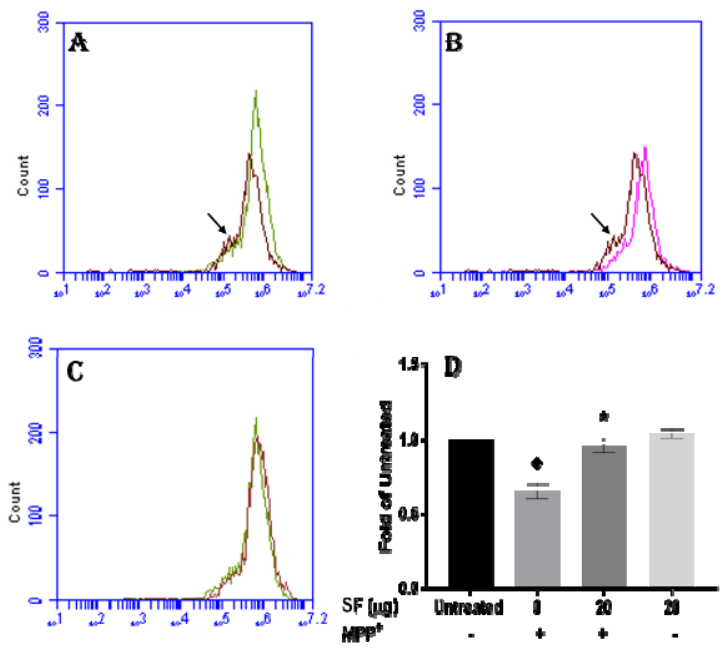

Figure 4: Effects of treatment with SF extract on $\mathrm{MPP}^{+}$-induced loss of MMP. (A) Untreated vs $\mathrm{MPP}^{+}$ alone (B) SF pretreated vs $\mathrm{MPP}^{+}$alone (C) Untreated vs SF alone (D) Graph showing the effect of SF on MMP. Arrows indicate fluorescence intensity of cells treated with $\mathrm{MPP}^{+}$only. $\$ p<0.005$ vs untreated $\mathrm{SH}-$ SY5Y cells; ${ }^{*} p<0.005$ vs SH-SY5Y cells treated with $\mathrm{MPP}^{+}$only

The reduction of rhodamine 123 fluorescence intensity signifies a loss of MMP. The graphs in Figure 4 showed that $\mathrm{MPP}^{+}$treatment alone significantly reduced $(p=0.0011)$ the MMP as demonstrated by a shift to the left in the fluorescence intensity while treatment with the SF extract appeared to inhibit $(p=0.0025)$ the $\mathrm{MPP}^{+}$-induced reduction in MMP. On the other hand, treatment with SF alone did not significantly affect ( $p=0.8877)$ the MMP when compared to untreated SH-SY5Y cells.

\section{Caspase $3 / 7$ and 9 activities following SF pretreatment}

The SH-SY5Y cells treated with $\mathrm{MPP}^{+}$alone showed that caspase $3 / 7(p=0.0006)$ and caspase $9(p=0.0011)$ activities were significantly increased, which is an indication of the activation of apoptosis (Figure 5). In cells pretreated with SF, there was a significant decrease in the activities of caspase $3 / 7(p=$ $0.0199)$ and caspase $9(p=0.0359)$ respectively. Treatment with SF alone did not significantly affect caspase $3 / 7(p=0.9184)$ and $9(p=$ 0.9917 ) activity when compared to the untreated SH-SY5Y cells.
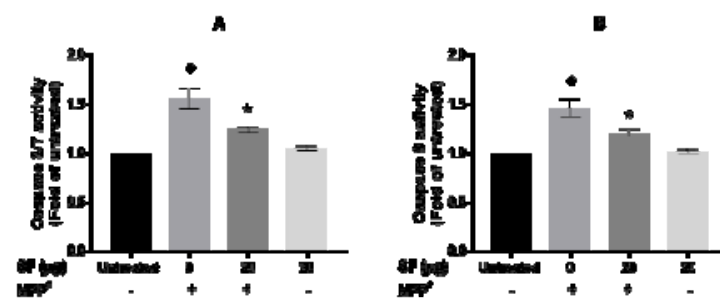

Figure 5: Effects of treatment with SF extract on MPP $^{+}$-induced apoptosis. (A) Caspase $3 / 7$ activity (B) Caspase 9 activity. $\bullet p<0.005$ vs untreated SH-SY5Y cells; ${ }^{*} p<0.05$ vs SH-SY5Y cells treated with MPP ${ }^{+}$ only

\section{DISCUSSION}

This study demonstrated the neuroprotective activity of SF in an in vitro model of PD using the neurotoxin $\mathrm{MPP}^{+}$and dopaminergic $\mathrm{SH}-\mathrm{SY} 5 \mathrm{Y}$ human neuroblastoma cells. Exposure of $\mathrm{SH}$ SY5Y cells to $\mathrm{MPP}^{+}$for $24 \mathrm{~h}$ significantly decreased cell viability, increased toxicity, increased intracellular ROS production, induced loss of MMP and activated caspases $3 / 7$ and 9 . However, the changes induced by $\mathrm{MPP}^{+}$were significantly inhibited by pretreatment of the $\mathrm{SH}$ SY5Y cells with SF, which suggests that SF may protect the cells from MPP ${ }^{+}$-induced apoptosis and loss of MMP via the regulation of ROS.

Previous studies have indicated that in PD patients, about $50 \%$ of dopaminergic neurons in the nigrostriatal pathway have degenerated in the brain at the time of preliminary diagnosis, and 
a substantial portion of the remaining dopaminergic neurons are stressed $[15,16]$. In a bid to replicate this condition in this model of PD, it was imperative to utilize concentrations of toxins that induced approximately $50 \%$ cell death. Findings from the MTT assay showed that the selected dose of $\mathrm{MPP}^{+}$treatment induced approximately $50 \%$ cell death, which is in line with earlier studies indicating that treatment of cells to high concentrations of $\mathrm{MPP}^{+}$for $24 \mathrm{~h}$ result in a mix of healthy and unhealthy cell population $[17,18]$. Findings from this study also show that pretreatment of SH-SY5Y cells with SF protected the cells from $\mathrm{MPP}^{+}$-induced toxicity. From the morphological images, it was observed that the shrinkage of cell bodies and the disappearance of axons were evident in the $\mathrm{MPP}^{+}$-treated cells, whereas cells pretreated with the SF extract looked similar to the untreated cells.

The pathogenesis of PD has been reported to involve excessive production of ROS and the ability of many medicinal plant extracts to reverse or modulate excess ROS is a known neuroprotection mechanism. $\mathrm{MPP}^{+}$is a known ROS-inducing neurotoxin, and overproduction of ROS has been reported to contribute to oxidative damage, mitochondrial dysfunction and apoptotic cell death [19]. One of the mechanisms reported for the excessive production of intracellular ROS by $\mathrm{MPP}^{+}$is the inhibition of the mitochondrial electron transport chain complex I [20]. This excessive production of intracellular ROS is believed to be an early event preceding the activation of caspase- 9 as well as caspase-3/7, leading to apoptosis [21]. These events show that ROS is a key player in cellular apoptosis induced by $\mathrm{MPP}^{+}$. The results further revealed that intracellular ROS production significantly increased following treatment of SH-SY5Y cells with $\mathrm{MPP}^{+}$. Conversely, intracellular ROS production significantly reduced when SH-SY5Y cells were pretreated with SF before $\mathrm{MPP}^{+}$ treatment.

Mitochondria are susceptible to oxidative stress and when there is sustained generation of ROS, there may be alterations in mitochondrial morphology, which further results in the opening of the mitochondrial transition pore, and a loss of MMP [19]. A reduction in membrane potential is an indicator of early apoptosis, and is consistent with previous findings. The results from the current study showed that SH-SY5Y cells displayed a decline in mitochondrial membrane potential upon treatment with $\mathrm{MPP}^{+}$. Conversely, pretreatment with SF prevented the loss of MMP which may suggest a neuroprotective effect of $\mathrm{SF}$ against $\mathrm{MPP}^{+}$-induced toxicity.
In the pathogenesis of PD, both the extrinsic (death-receptor) and the intrinsic (or mitochondrial) apoptotic pathways are known to play major roles. Investigations on the events involved in the intrinsic pathway show that cytochrome $\mathrm{c}$ binds to the apoptosis protease activating factor 1 (Apaf-1) in the cytoplasm to form an apoptosome (a molecular complex consisting of cytochrome c, Apaf-1, ATP, and procaspase 9). The apoptosome in turn activates caspase 9 (an upstream initiator of apoptosis) which then activates the executioner caspases 3 , 6 and 7 , eventually resulting in apoptosis [22]. In the present study, the activity of caspases $3 / 7$ and 9 significantly increased in SH-SY5Y cells treated with $\mathrm{MPP}^{+}$alone. However, pretreatment of cells with SF significantly inhibited the activity of these caspases, indicating that SF inhibited the activation of the intrinsic apoptotic pathway by $\mathrm{MPP}^{+}$.

\section{CONCLUSION}

The findings of the current study demonstrate the neuroprotective activity of the SF extracts against $\mathrm{MPP}^{+}$-induced toxicity in SH-SY5Y cells. The SF extracts appeared to inhibit $\mathrm{MPP}^{+}$-induced overproduction of ROS as well as a loss of MMP. These extracts could attenuate the activation of the intrinsic apoptotic pathway, thus suggesting the potential use of the plant as a neuroprotective and therapeutic agent against PD.

\section{DECLARATIONS}

\section{Conflict of interest}

No conflict of interest is associated with this work.

\section{Contribution of authors}

We declare that this work was done by the authors named in this article and all liabilities pertaining to claims relating to the content of this article will be borne by the authors. A.B.E. and S.I.O. performed the experiments and wrote manuscript. OEE designed the experiments and edited the manuscript.

\section{Open Access}

This is an Open Access article that uses a funding model which does not charge readers or their institutions for access and distributed under the terms of the Creative Commons Attribution License (http://creativecommons.org/licenses/by/ 4.0) and the Budapest Open Access Initiative 
(http://www.budapestopenaccessinitiative.org/rea d), which permit unrestricted use, distribution, and reproduction in any medium, provided the original work is properly credited.

\section{REFERENCES}

1. Park A, Stacy M. Non-motor symptoms in Parkinson's disease. J Neurol. 2009; 256(3): 293-298.

2. Przedborski S, Jackson-Lewis V. Mechanisms of MPTP toxicity. Mov Disord. 1998; 13: 35-38.

3. Shibata $N$, Kobayashi $M$. The role for oxidative stress in neurodegenerative diseases. Brain Nerve. 2008; 60(2): 157-170.

4. Niranjan $R$. The role of inflammatory and oxidative stress mechanisms in the pathogenesis of Parkinson's disease: focus on astrocytes. Mol Neurobiol. 2014; 49(1): 28-38.

5. Keeney PM, Xie J, Capaldi RA, Bennett JP. Parkinson's disease brain mitochondrial complex I has oxidatively damaged subunits and is functionally impaired and misassembled. J Neurosci. 2006; 26(19): 5256-5264.

6. Levy OA, Malagelada C, Greene LA. Cell death pathways in Parkinson's disease: proximal triggers, distal effectors, and final steps. Apoptosis. 2009; 14(4): 478500 .

7. Gericke N, Albrecht C, Van Wyk B, Mayeng B, Mutwa C, Hutchings A. Sutherlandia frutescens. AJMH. 2001; 13(1): 9-15.

8. Van Wyk B, Albrecht C. A review of the taxonomy, ethnobotany, chemistry and pharmacology of Sutherlandia frutescens (Fabaceae). J Ethnopharmacol. 2008; 119(3): 620-629.

9. Gericke N. Sutherlandia and AIDS patients: update 13 March 2001. AJMH. 2001; 13(1): 17.

10. Skerman NB, Joubert AM, Cronjé MJ. The apoptosis inducing effects of Sutherlandia spp. extracts on an oesophageal cancer cell line. J Ethnopharmacol. 2011; 137(3): 1250-1260.

11. MacKenzie J, Koekemoer TC, Roux S, van de Venter M, Dealtry GB. Effect of Sutherlandia frutescens on the lipid metabolism in an insulin resistant rat model and 3T3-L1 adipocytes. Phytother Res. 2012; 26(12): 1830-1837.

12. Ojewole J. Analgesic, antiinflammatory and hypoglycemic effects of Sutherlandia frutescens $R$. BR. (variety Incana
E. MEY.) (Fabaceae) shoot aqueous extract. Methods Find Exp Clin Pharmacol. 2004; 26(6): 409-416.

13. Van de Loosdrecht A, Beelen R, Ossenkoppele G, Broekhoven M, Langenhuijsen $M$. A tetrazolium-based colorimetric MTT assay to quantitate human monocyte mediated cytotoxicity against leukemic cells from cell lines and patients with acute myeloid leukemia. J Immunol Methods. 1994; 174(1-2): 311-320.

14. Slater $T$, Sawyer B, Sträuli U. Studies on succinatetetrazolium reductase systems: III. Points of coupling of four different tetrazolium salts III. Points of coupling of four different tetrazolium salts. Biochim Biophys Acta. 1963; 77: 383-393.

15. Collier TJ, Kanaan NM, Kordower JH. Ageing as a primary risk factor for Parkinson's disease: evidence from studies of non-human primates. Nature Reviews Neuroscience. 2011; 12(6): 359.

16. Bernheimer H, Birkmayer W, Hornykiewicz O, Jellinger K, Seitelberger F. Brain dopamine and the syndromes of Parkinson and Huntington Clinical, morphological and neurochemical correlations. J Neurol Sci. 1973; 20(4): 415-455.

17. Gong P, Deng F, Zhang W, Ji J, Liu J, Sun Y, Hu J. Tectorigenin attenuates the MPP+-induced SH-SY5Y cell damage, indicating a potential beneficial role in Parkinson's disease by oxidative stress inhibition. Exp Ther Med. 2017; 14(5): 4431-4437.

18. Janhom P, Dharmasaroja P. Neuroprotective Effects of Alpha-Mangostin on MPP. J Toxicol. 2015; 2015.

19. Cassarino DS, Parks JK, Parker Jr WD, Bennett Jr JP. The parkinsonian neurotoxin MPP+ opens the mitochondrial permeability transition pore and releases cytochrome $c$ in isolated mitochondria via an oxidative mechanism. Biochim Biophys Acta, Mol Basis Dis. 1999; 1453(1): 49-62.

20. Nicklas W, Vyas I, Heikkila RE. Inhibition of NADH-linked oxidation in brain mitochondria by 1-methyl-4-phenylpyridine, a metabolite of the neurotoxin, 1-methyl-4phenyl-1, 2, 5, 6-tetrahydropyridine. Life Sci. 1985; 36(26): 2503-2508.

21. Venderova K, Park DS. Programmed cell death in Parkinson's disease. Cold Spring Harb Perspect Med. 2012; 2(8): a009365.

22. Liu X, Kim CN, Yang J, Jemmerson R, Wang X. Induction of apoptotic program in cell-free extracts: requirement for dATP and cytochrome c. Cell. 1996; 86(1): 147-157. 